20. British Society of Audiology, Occupational hearing loss, 2004; http://www.occupationalhearingloss.com

21. Kavanagh, K. T., Evaluation of occupational hearing loss and presbycusis using a micro-computer. J. Am. Acad. Audiol., 1992, 3, 215-220.

22. Kavanagh, K. T., Evaluation of hearing handicaps and presbycusis using world wide web based calculators. J. Am. Acad. Audiol., 2001, 12, 497-505.

ACKNOWLEDGEMENTS. We thank the Director, ICAR-Central Institute of Agricultural Engineering, Bhopal and Dean, Peoples College of Medical Sciences and Research Centre, Bhopal for their support and for providing the necessary facilities to conduct this study. We also thank the staff of the Department of ENT, Peoples College of Medical Sciences and Research Centre, and all the subjects who took part in the study.

Received 25 May 2016; revised accepted 10 April 2017

doi: $10.18520 / \mathrm{cs} / \mathrm{v} 113 / \mathrm{i} 05 / 969-974$

\section{Face recognition using a hybrid SVM-LBP approach and the Indian movie face database}

\author{
Francisco A. Pujol*, Antonio Jimeno-Morenilla \\ and José Luis Sánchez-Romero \\ Computer Technology Department, Universidad de Alicante, \\ P.O. Box 99, 03080 Alicante (Spain)
}

Local binary patterns (LBP) are an effective texture descriptor for face recognition. In this work, a LBPbased hybrid system for face recognition is proposed. Thus, the dimensionality of LBP histograms is reduced by using principal component analysis and the classification is performed with support vector machines. The experiments were completed using the challenging Indian Movie Face Database and show that our method achieves high recognition rates while reducing $95 \%$ the dimensions of the original LBP histograms. Moreover, our algorithm is compared against some state-of-the-art approaches. The results indicate that our method outperforms other approaches, with accurate face recognition results.

Keywords: Face recognition, hybrid methods, local binary patterns, support vector machines.

\footnotetext{
*For correspondence. (e-mail: fpujol@dtic.ua.es)
}

BIOMETRIC recognition allows identification or verification of the identity from the user's unique morphological or behavioural characteristics. Among all the biometric systems, face recognition is one of the most common techniques to identify users. Local binary patterns (LBP) and its variants have been used successfully in face recognition during the last few years ${ }^{1}$. However, the length of generated feature vectors may cause a slow processing of face images.

The main contributions of our work are:

- The dimensions of the LBP vectors will be reduced by using PCA. Therefore, only the principal LBPs will be considered and the computation of the needed features will be accelerated.

- Next, a support vector machine (SVM) will be used so that it accurately identifies if the LBP belongs to the user to be recognized.

- The experiments are performed using the recent and challenging Indian Movie Face Database (IMFDB) ${ }^{2}$. There are few studies that deal with this database, due to its complexity and novelty ${ }^{3,4}$, and no previous reference has been found using the IMFDB with the LBP algorithm. Moreover, a comparison with related methods is included, as well. No comparison on the results of different face recognition methods with the IMFDB has been reported before either.

LBPs were introduced to describe textures in images, considering that a texture consists of two complementary characteristics, the threshold and the weight ${ }^{1}$. LBPs encode the relationship of the central pixel $g_{c}$ to the gray intensity of the pixels in the neighbourhood $g_{p}$. The value of the central pixel is taken as a threshold. Then, the LBP label for the central pixel $(x, y)$ for each neighbourhood of $P$ pixels is computed by multiplying the values of the threshold by the weights given to the corresponding pixels and, finally, the result is added

$$
\operatorname{LBP}_{P}(x, y)=\sum_{p=0}^{P-1} s\left(g_{p}-g_{c}\right) 2^{p},
$$

where $s(x)=\left\{\begin{array}{ll}1, & x \geq 0 \\ 0, & x<0\end{array}\right.$.

Ahonen $^{5}$ introduced LBPs for face recognition. Thus, after dividing the face image into $R$ uniform regions, the histograms of the computed LBP labels for each region, $H^{w}$, with $w=\{1,2, \ldots, R\}$ were concatenated to obtain the histogram representing a face. A LBP histogram is defined as

$$
H^{w}(i)=\sum_{x, y \in \text { block }_{w}} I(f(x, y)=i), i=1, \ldots, N,
$$

where $N$ is the number of bins, $f(x, y)$ the LBP label at pixel $(x, y)$ and $I$ is the indicator function ${ }^{5}$. 

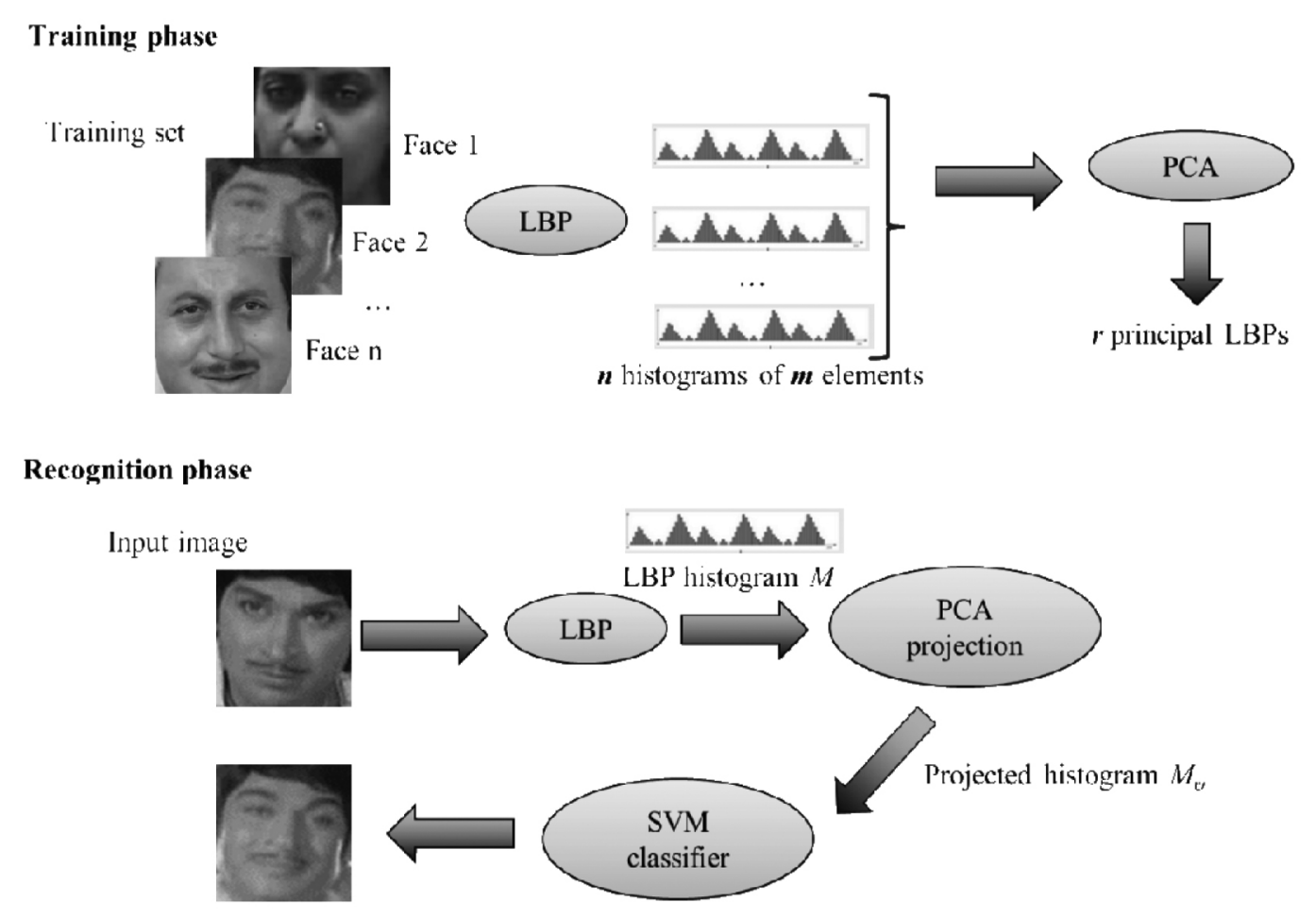

Recognized user

Figure 1. Overview of the hybrid recognition method.

In Ahonen's approach, face images were divided using a regular grid. In particular, a $7 \times 7$ mask was used and, as a result, images are divided into 49 regions to obtain the corresponding LBP histograms. Only 39 of these regions are actually important in face recognition, giving, as a result a vector with 2301 components in total per image. In general, using an arbitrary mask and a certain neighbourhood, a vector of $m$ features will be obtained.

If we consider that each image is split into the same number of regions, depending on the selected mask, any attempt to reduce the feature vector must involve reducing the number of LBPs in the image. One of the most popular ways to reduce the dimensions of the feature vectors is PCA. PCA takes a data matrix of $n$ objects by $m$ variables and calculates the so-called principal components, which are linear combinations of the original $m$ variables. The first $r$ components display the highest variation among faces. Thus, let $\left(H_{1}, H_{2}, \ldots, H_{n}\right)$ be a set of $n$ LBP histograms from $n$ training face images, arranged as a column vector.

As a result, the set of $r(r \ll n)$ eigenvectors of the covariance matrix $\sum$ with the $r$ largest eigen values will be selected. An $n \times r$ matrix $\Psi=\left[\psi_{1}, \psi_{2}, \ldots, \psi_{\mathrm{r}}\right]$ is composed to perform the PCA projection. Now, for any given new LBP histogram $S$, a corresponding point in the $r$-dimensional PCA space, $S_{\psi}$, can be found by computing

$$
S_{\psi}=(S-\phi) \cdot \Psi,
$$

where $\phi$ is the average LBP histogram.

Figure 1 shows an overview of our approach to reduce dimensionality, where the training and recognition phases are described.

In order to classify the resulting histograms, SVMs were used. The main advantage of SVM classification is that it performs well on datasets that have many attributes, even when there are only a few cases that are available for the training process. This fact is especially useful for face recognition.

SVMs construct a hyperplane as the decision plane, separating the positive $(+1)$ and negative $(-1)$ classes with the largest margin. Given the LBP histograms training set $S_{\psi_{i}} \in \boldsymbol{R}^{m}$ with two separate classes $y_{i} \in\{-1,1\}$, for $i=1, \ldots, r$, the algorithm maximizes the margin of error, which is the perpendicular distance from the separating $m$-dimensional hyperplane $\boldsymbol{w}: \boldsymbol{w} \cdot \boldsymbol{S}_{\psi}+b=0$

$$
\max _{w, b} \frac{1}{\|\boldsymbol{w}\|}
$$

subject to

$$
y_{i}\left(\boldsymbol{w}^{T} S_{\psi_{i}}-b\right) \geq 1, i=1, \ldots, r .
$$



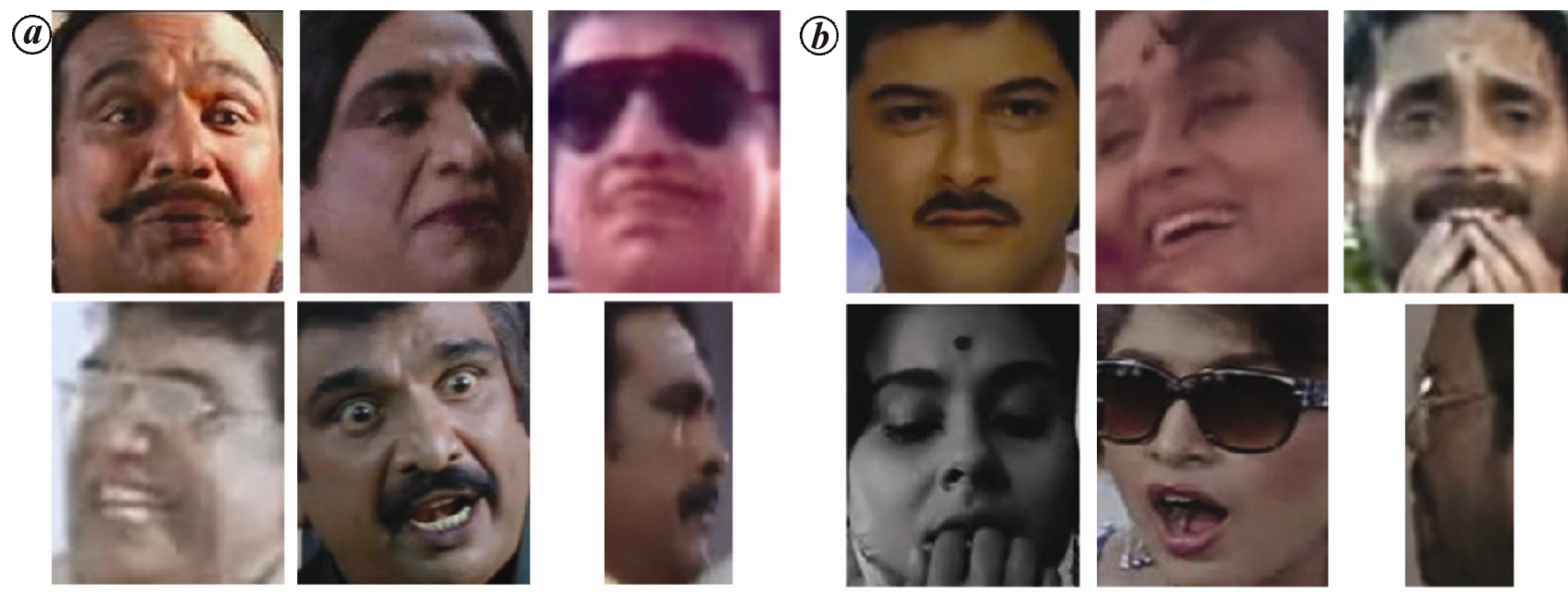

Figure 2. Some images from the IMFDB. $\boldsymbol{a}$, Images from the same person. $\boldsymbol{b}$, Images from different persons.

Our system aims at finding a classifier $f\left(M_{\psi}\right)$ for the projected LBP histogram $M_{\psi}$ from a new input image. The class $y$ is determined by the sign of the decision function

$$
f\left(M_{\psi}\right)=\boldsymbol{w}^{T} \phi\left(M_{\psi}\right)+b=\sum_{j=1}^{k} \alpha_{j} y_{j} K\left(M_{\psi}, S_{\psi_{j}}\right)+b
$$

where $\alpha_{j}$ are positive real constants and $K\left(M_{\psi}, S_{\psi_{j}}\right)$ is the kernel function.

As the system will identify a number of users $q$, a multi-class SVM will be used. Thus, $q$ SVM classifiers $f^{1}, f^{2}, \ldots, f^{q}$, following eq. (6), are formed and trained. At the classification phase, a new input image, i.e. a new LBP projected histogram $M_{\psi}$, is classified as in class $i$ whose $f^{i}$ has the largest value

$$
i=\underset{i=1, \ldots, q}{\arg \max } f^{i}\left(M_{\psi}\right)=\underset{i=1, \ldots, q}{\arg \max } \boldsymbol{w}_{i}^{T} \phi\left(M_{\psi}\right)+b_{i} .
$$

Our proposal has been experimented with IMFDB ${ }^{1}$. Some sample images are shown in Figure 2. IMFDB is a huge face database with 34,512 images of Indian actors extracted from Indian movies. In this database, images were manually processed from original video frames, resulting in a highly challenging dataset. For every image, the following features are given: expression, illumination, pose, occlusion, age, makeup and gender. In our experiments, images were divided into 4 subsets. Subset 1 included only frontal images with good illumination conditions and no occlusions. Subset 2 included frontal images with no occlusions and medium or bad lighting situations. Then, subset 3 contained images with changes in pose, any illumination conditions and no occlusions. Finally, subset 4 was composed by images not belonging to any of the other subsets.
Two frontal images per subject from subset 1 were used for the training set. The remaining images were considered as probes. All images were rescaled to $60 \times 60$ pixels and converted to grey scale. The tests were performed using Matlab ${ }^{\circledR}$ with a $3.1 \mathrm{GHz}$ Intel Core i5 and 16 GB memory. Finally, to compare with Ahonen's proposal, a $7 \times 7$ mask for the LBPs was chosen.

The first set of experiments was addressed to determine the parameters to achieve a robust face recognition. As mentioned before, the training process was performed with two frontal faces per individual, i.e. 200 images. The parameters to be chosen were the number of principal LBPs $r$, the neighbourhood of $\operatorname{LBP}(P, R)$ and the kernel function for SVM. When calculating the recognition rate for one parameter, the others were fixed, so that depending on the test the number of eigenvectors was 20 , a radial basis function (RBF) kernel was selected and a neighbourhood $(8,4)$ was used for the LBPs. Figure 3 graphically shows the mean recognition rates obtained from the experiments.

After analysing the results, it was observed that from $r=20$ eigen vectors, the correct recognition is virtually constant (see Figure $3 a$ ). As a result, let us consider $r=20$ as the amount of principal LBPs for our recognition scheme. Therefore, since the system originally had 400 LBP vectors for the training database (2 per person), PCA reduces almost $95 \%$ the dimensions of the training database with a good recognition rate of $90.5 \%$. Moreover, it has been shown that the RBF kernel (Figure $3 b$ ) has the best recognition rates compared with other kernels and that a neighbourhood of $(8,4)$ to compute the LBPs achieves prominent results (Figure $3 c$ ).

Our method was then compared with other state-of-theart algorithms for face recognition. The recognition results for these methods can be found in Table 1. As shown, since image conditions for subset 1 are satisfactory, all the methods achieve good recognition results. Then, 
RESEARCH COMMUNICATIONS

Table 1. Comparison with other methods

\begin{tabular}{|c|c|c|c|c|c|}
\hline \multirow[b]{2}{*}{ Method } & \multicolumn{5}{|c|}{ Average recognition rate $(\%)$} \\
\hline & Subset 1 & Subset 2 & Subset 3 & Subset 4 & Mean \\
\hline $\operatorname{LBP}(8,4)^{5}$ & 97.1 & 88.6 & 76.1 & 56.1 & 72.7 \\
\hline $\mathrm{LGBP}^{6}$ & 94.3 & 97.2 & 78.7 & 58.7 & 76.0 \\
\hline $\mathrm{DLBP}^{7}$ & 98.3 & 97.9 & 80.6 & 62.6 & 78.6 \\
\hline SLF-RKR ${ }^{8}$ & 99.2 & 98.7 & 81.2 & 61.2 & 78.5 \\
\hline POEM $^{9}$ & 98.2 & 89.4 & 79.8 & 59.8 & 75.6 \\
\hline Our approach & 99.2 & 98.9 & 83.4 & 63.4 & 80.1 \\
\hline
\end{tabular}

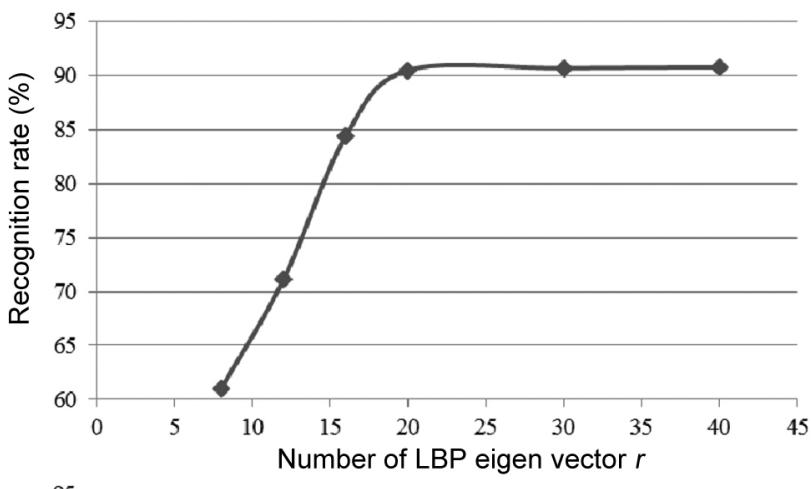

images. Finally, when using subset 4 , the results are not good in general terms: the high variability in the images of this subset makes it difficult to achieve high recognition rates.

From these results, it must be pointed out that our approach out-performs the others in most cases, even when using the challenging subset 4. Moreover, our method reduces almost $95 \%$ the dimension of the LBP vectors in the database. Subsequently, it has been proved that our LBP-based algorithm is a suitable option for face recognition.
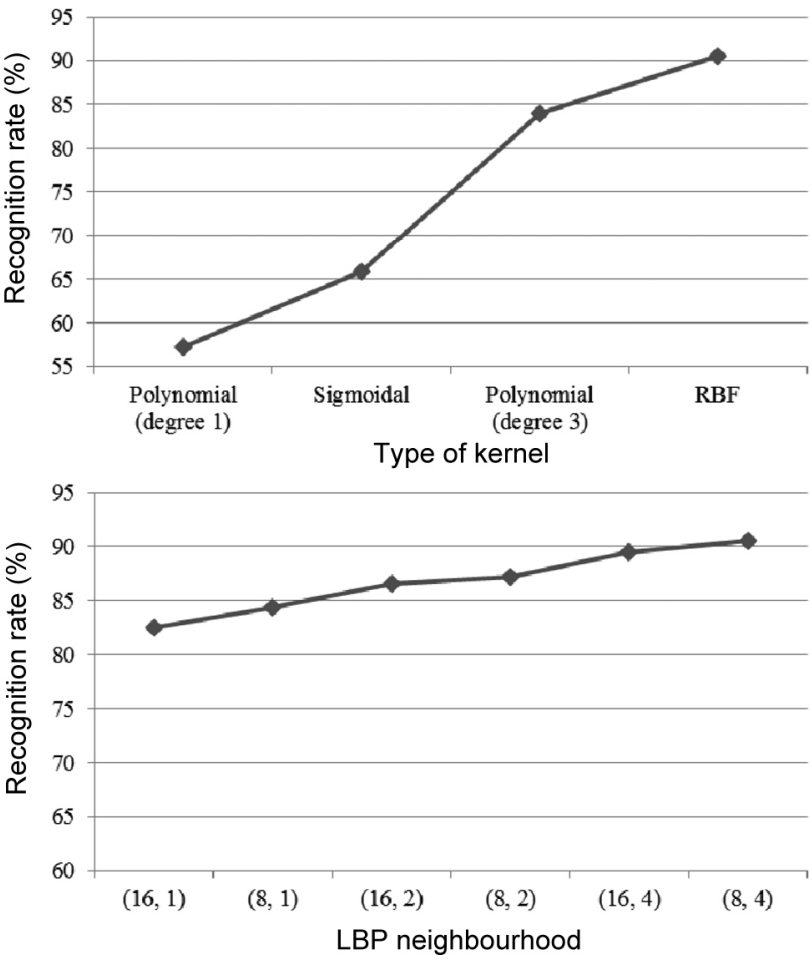

1. Ojala, T., Pietikäinen, M. and Harwood, D., A comparative study of texture measures with classification based on featured distributions. Pattern Recogn., 1996, 29, 51-59.

2. Setty, S. et al., Indian movie face database: a benchmark for face recognition under wide variations. In Proceedings of the 2013 Fourth National Conference on Computer Vision, Pattern Recognition, Image Processing and Graphics (NCVPRIPG), 2013.

3. Beham, M. P., Roomi, S. and Kapileshwaran, V., Robust face recognition using automatic pose clustering and pose estimation. In Proceedings of the 2013 Fifth International Conference on Advanced Computing (ICoAC), 2013.

4. Kumar, V., Namboodiri, A.M. and Jawahar, C.V., Face recognition in videos by label propagation. In Proceedings of the 22nd International Conference on Pattern Recognition (ICPR), 2014.

5. Ahonen, T., Hadid, A. and Pietikäinen, M., Face recognition with local binary patterns. In Proceedings of the 8th European Conference on Computer Vision, ECCV 2004, Prague, Czech Republic, 2004, pp. 469-481.

6. Zhang, W., Shan, S., Gao, W., Chen, X. and Zhang, H., Local Gabor binary pattern histogram sequence (lgbphs): a novel non-statistical model for face representation and recognition. In Proceedings of the 10th International Conference on Computer Vision, 2005.

7. Maturana, D., Mery, D. and Soto, A., Learning discriminative local binary patterns for face recognition. In Proceedings of the IEEE International Conf. on Automatic Face \& Gesture Recognition, 2011.

8. Yang, M., Zhang, L., Shiu, S. K. and Zhang, D., Robust kernel representation with statistical local features for face recognition. IEEE $T$. Neural Network., 2013, 24, 900-912.

Figure 3. $\boldsymbol{a}$, Recognition rates for different number of LBP eigenvec tors. $\boldsymbol{b}$, Recognition rates for different SVM kernels. $\boldsymbol{c}$, Recognition rates when using different neighbourhoods to compute the LBPs.

changes in lighting conditions (subset 2) do not affect the recognition process for the algorithms considered. When pose changes (subset 3), the performance of algorithms decreases, mainly due to out-of-plane rotations for some

9. Vu, N. S. and Caplier, A., Enhanced patterns of oriented edge magnitudes for face recognition and image matching. IEEE T. Image Process., 2012, 21, 1352-1365.

Received 13 October 2016; revised accepted 28 April 2017

doi: $10.18520 / \mathrm{cs} / \mathrm{v} 113 / \mathrm{i} 05 / 974-977$ 